Ingrid Teixeira Monteiro

\title{
Autoexpressão e engenharia semiótica do usuário-designer
}

Tese de Doutorado

Tese apresentada ao Programa de Pósgraduação em Informática do Departamento de Informática do Centro Técnico Científico da PUCRio, como requisito parcial para a obtenção do grau de Doutor em Informática.

Orientadora: Prof. a . Clarisse Sieckenius de Souza 
Ingrid Teixeira Monteiro

\title{
Autoexpressão e engenharia semiótica do usuário-designer
}

Tese apresentada ao Programa de Pós-graduação em Informática do Departamento de Informática do Centro Técnico Científico da PUC-Rio, como requisito parcial para a obtenção do grau de Doutor em Informática. Aprovada pela Comissão Examinadora abaixo assinada.

\author{
Prof.a. Clarisse Sieckenius de Souza \\ Orientador \\ Departamento de Informática - PUC-Rio
}

Prof. ${ }^{\text {. }}$ Milene Selbach Silveira

PUC-RS

Prof. Vinícius Carvalho Pereira UFMT
Prof. a. Carla Faria Leitão
PUC-Rio

Prof. Alberto Barbosa Raposo

PUC-Rio

Prof. José Eugenio Leal Coordenador Setorial do Centro Técnico Científico PUC-Rio 
Todos os direitos reservados. É proibida a reprodução total ou parcial do trabalho sem autorização da universidade, da autora e do orientador.

\section{Ingrid Teixeira Monteiro}

Graduou-se em Telemática - Telecomunicações pelo Centro Federal de Educação Tecnológica do Ceará (2007) e em Comunicação Social - Publicidade e Propaganda pela Universidade Federal do Ceará (2006). Possui mestrado em Informática na área de Interação HumanoComputador pela Pontifícia Universidade Católica do Rio de Janeiro (2011).

Ficha Catalográfica

Monteiro, Ingrid Teixeira

Autoexpressão e engenharia semiótica do usuário-designer / Ingrid Teixeira Monteiro; orientador:

Clarisse Sieckenius de Souza. - 2015.

312 f. : il. (color.) ; $30 \mathrm{~cm}$

Tese (doutorado) - Pontifícia Universidade Católica do Rio de Janeiro, Departamento de Informática, 2015.

Inclui bibliografia

1. Informática - Teses. 2. Engenharia Semiótica. 3. End-User Development. 4. Autoexpressão. 5. Computador como mídia. 6. SideTalk. I. Souza, Clarisse Sieckenius de. II. Pontifícia Universidade Católica do Rio de Janeiro. Departamento de Informática. III. Título. 


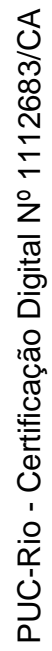

À minha grande família Teixeira. 


\section{Agradecimentos}

Uma tese não é apenas o trabalho de um doutorando e de seu orientador. Esta, em particular, tem as mãos de várias pessoas, não apenas em relação ao texto aqui apresentado, mas também em relação ao percurso nestes quatro anos, envolvendo disciplinas cursadas, execução de estudos empíricos, discussões em grupo e principalmente paciência e compreensão daqueles mais próximo que, com todo amor, nos perdoam sempre pelas ausências típicas de um doutorado.

Agradeço primeiramente à minha orientadora, Clarisse Sieckenius de Souza, que desde o começo do meu mestrado tem me ensinado o que significa "fazer pesquisa". Toda a sua competência, experiência e inteligência permitiram que construíssemos juntas um trabalho interessante e de qualidade. Devo a ela também todo apoio e compreensão demonstrados a cada decisão importante que eu tomei ao longo do doutorado.

Deixo meu agradecimento aos membros da banca, por terem aceitado ler essa tese enorme e por me ajudarem a melhorar o trabalho a partir de suas contribuições durante a defesa.

Agradeço aos colegas queridos que me acompanharam neste caminho longo e árduo, mas recompensador. Marcelle, Juliana e Cleyton foram os companheiros mais próximos. Trabalhamos muito, mas também nos divertimos um bocado. A eles e aos demais colegas do SERG (Carla, Luciana, Priscilla, Luiz, Rafael, João e Bruno) devo o interesse, as contribuições e as muitas horas de atenção durante os seminários semanais que tanto enriqueceram nossos conhecimentos, com discussões e contribuições interessantes e valiosas.

Aos membros do projeto SGD-Br agradeço pela incrível oportunidade de trabalhar intensivamente numa iniciativa tão bacana e inspiradora como esta de ensinar crianças a programar.

Agradeço especialmente aos professores das escolas parceiras do projeto, à IBM Research, à Marcelle, ao João, à Aline, à Viviane que, de uma forma ou de outra, contribuíram para a realização dos estudos empíricos que deram corpo a esta tese. Destaco a contribuição do Eduardo não apenas na realização dos estudos mas também no desenvolvimento do SideTalk ao longo de mais de dois anos de parceria. Não poderia deixar de mencionar também minha enorme gratidão a todos os participantes por sua colaboração, entusiasmo e generosidade.

Devo um agradecimento também aos professores da PUC que contribuíram para a minha formação de pesquisadora, bem como ao CNPq pelo apoio financeiro. 
Aos que se mantiveram geograficamente distantes, agradeço a imensa torcida e confiança dos meus queridos amigos de Fortaleza. À minha família, especialmente mãe e irmãs, agradeço o apoio incondicional dado não apenas durante os últimos quatro anos, mas ao longo de toda a minha jornada.

À minha enteada Luna e ao meu marido Emmanuel devo não apenas agradecer a compreensão e paciência, mas principalmente me desculpar por todo o tempo que deixei de estar perto, todos os programas abdicados e todo o mau-humor que vez outra eles tiveram que aguentar.

Especialmente no período em que eu estive no Rio de Janeiro para trabalhar na tese, agradeço à D. Maria e Clarice, por terem me recebido com tanta boa-vontade em sua casa, à Lurdinha por ter cuidado da Luna e da minha casa; e ao João, Luana e Cleyton pela companhia no SERG. Ao Cleyton também registro minha gratidão a todo o suporte dado na fase de certificação, impressão e entrega do texto final da tese.

Um agradecimento atípico, mas sincero, vai para a minha gatinha Sakura, que literalmente ficou perto de mim (mais especificamente em cima da minha mesa) por horas e horas durante a escrita da tese. Suas poses, caras e bocas enquanto dormia e sua "autoridade" ao desfilar na frente do monitor, ao invés de atrapalhar, me ajudaram a relaxar um pouco durante esse período tão tenso.

Finalmente, agradeço à minha mais nova família: colegas, professores e alunos da Universidade Federal do Ceará (Quixadá) que me acolheram tão bem e que têm mostrado tanto apoio e torcida nessa reta final. Agradeço especialmente à Andreia que me incentivou a fazer o concurso que me permitiu "voltar pra casa". 


\section{Resumo}

Monteiro, Ingrid Teixeira; de Souza, Clarisse Sieckenius. Autoexpressão e engenharia semiótica do usuário-designer. Rio de Janeiro, 2015. 312p. Tese de Doutorado - Departamento de Informática, Pontifícia Universidade Católica do Rio de Janeiro.

A tese apresenta uma pesquisa inserida na área de End-User Development (EUD). As primeiras iniciativas nesta área surgiram como uma tentativa de auxiliar os usuários finais a atingirem objetivos específicos de personalização e customização de interfaces e sistemas, prioritariamente para benefício próprio. Conforme as necessidades evoluem, os usuários finais precisam conhecer e muitas vezes dominar conceitos e práticas mais complexos de computação. Neste contexto, têm surgido várias iniciativas para incentivar, ensinar e apoiar estes usuários a programarem e a pensarem computacionalmente. Geralmente, muita ênfase é dada para a questão da resolução de problemas, raciocínio lógico e outras habilidades comuns a cientistas da computação. Entretanto, apoiados na Engenharia Semiótica, uma teoria de base semiótica que descreve a interação humano-computador como uma comunicação entre designers e usuários, consideramos que sistemas computacionais interativos são artefatos comunicacionais: quem cria o sistema transmite mensagens, com características particulares, a quem os utiliza. Nesta tese, apresentamos um estudo em profundidade em que usuários finais, atuando como designers, criaram artefatos computacionais para fins de comunicação. A pesquisa mostrou que os participantes tomaram a programação e outras atividades de desenvolvimento não como fins em si, mas como meios para construírem suas mensagens. Discutimos como a mudança de perspectiva (de resolução de problemas para comunicação) revela fenômenos ainda pouco explorados como a autoexpressão dos designers nestes artefatos e a pragmática da linguagem de interação por eles construída. Outra contribuição da tese é uma extensão da Engenharia Semiótica, batizada de EUME - End-User Semiotic Engineering, uma forma de ver a Engenharia Semiótica na perspectiva do usuário final que atua como designer.

\section{Palavras-chave}

Engenharia Semiótica; End-User Development; Autoexpressão; Computador como mídia; SideTalk. 


\section{Abstract}

Monteiro, Ingrid Teixeira; de Souza, Clarisse Sieckenius (Advisor). Userdesigner's self-expression and semiotic engineering. Rio de Janeiro, 2015. 312p. Ph.D Thesis - Departamento de Informática, Pontifícia Universidade Católica do Rio de Janeiro.

This thesis presents research in the area the area of End-User Development (EUD). The first studies in EUD have emerged as an attempt to help end users achieve specific goals of personalization and customization of interfaces and systems, primarily for their own benefit. As needs evolve, end users have to know and often master more complex computing concepts and practices. In this context, there have been a growing number of initiatives to encourage, teach and support users in programming and thinking computationally. In general, much emphasis is given to problem solving, logical reasoning and other common computer scientists' skills. However, supported by Semiotic Engineering, a semiotics-based theory that describes human-computer interaction as communication between designers and users, we believe that interactive computer systems are communication artifacts: that the person who creates the system sends various messages, with particular characteristics to the person who uses it. In this thesis, we present an extensive study in which end users, acting as designers, create computational artifacts for communication purposes. Research has shown that the participants took programming and other development activities not as end in themselves but as a means to build their messages. We discuss how the change in perspective (from problem-solving to communication) reveals a range of underexplored phenomena, such as self-expression of the designers and the pragmatics of interaction languages they build. Another contribution of this thesis is an extension to Semiotic Engineering, named EUME - End-User Semiotic Engineering, a new way to look at Semiotic Engineering, in the perspective of end users acting as designers.

\section{Keywords}

Semiotic Engineering; End-User Development; Self-expression; Computer as media; SideTalk. 


\section{Sumário}

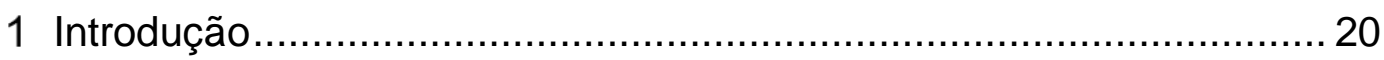

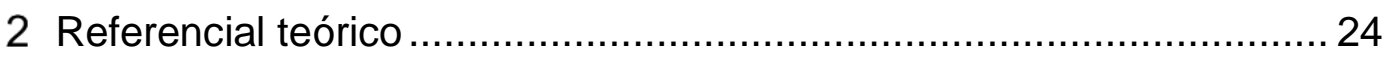

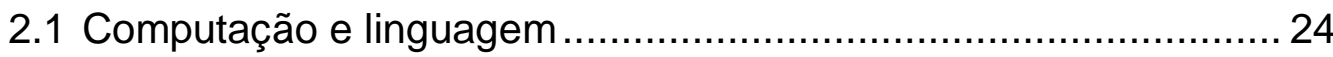

2.2 Programação e raciocínio computacional ....................................... 25

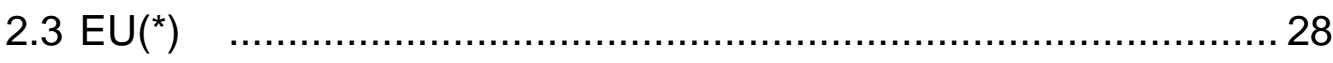

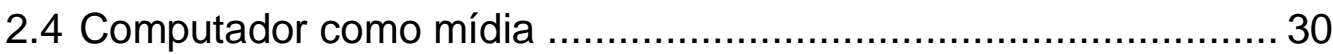

2.5 Engenharia Semiótica............................................................ 31

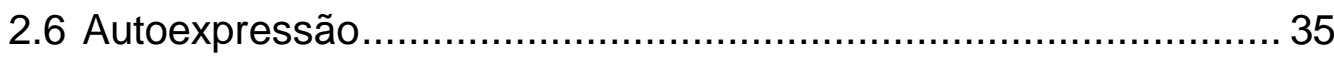

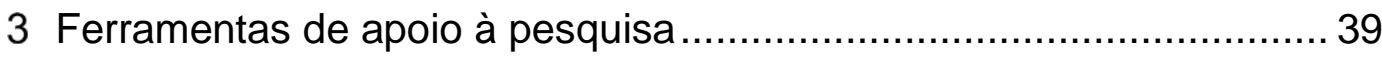

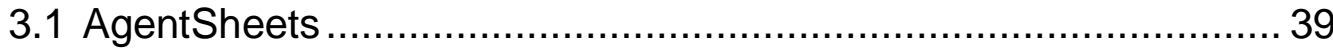

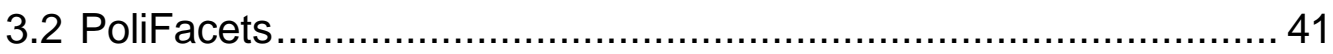

3.2.1 Faceta descrição ..................................................... 43

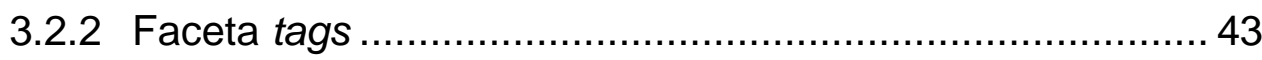

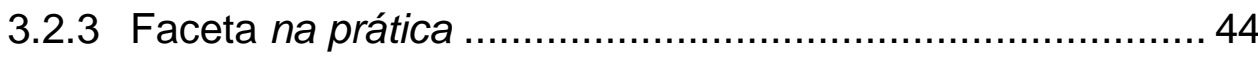

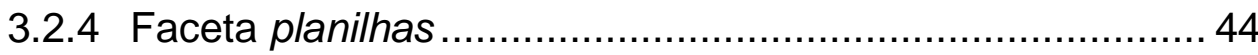

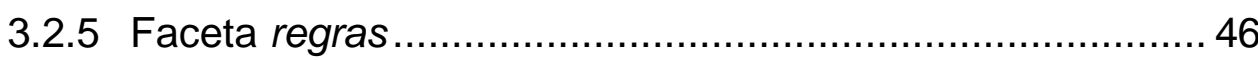

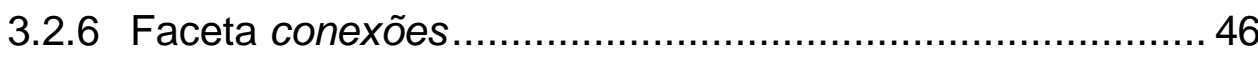

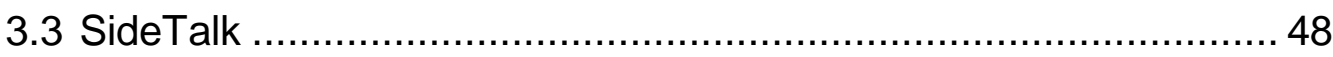

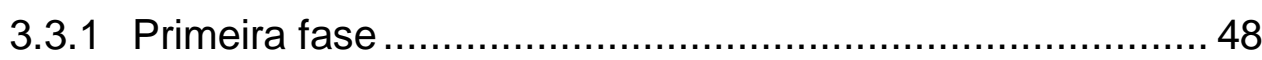

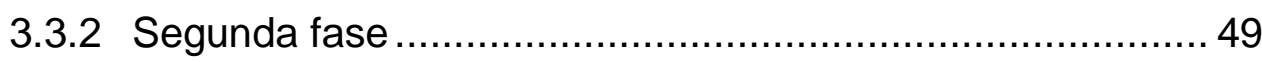

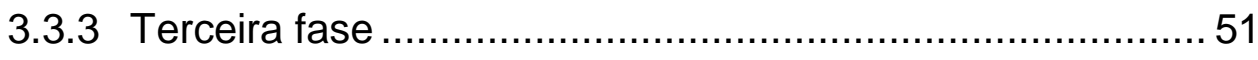

3.3.4 Criação de conversas mediadas ........................................ 56

3.3.5 Engenharia Semiótica no SideTalk..................................... 60

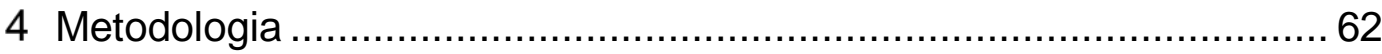

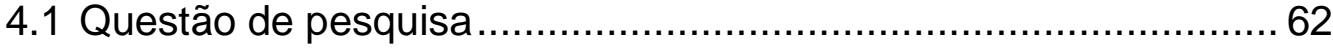

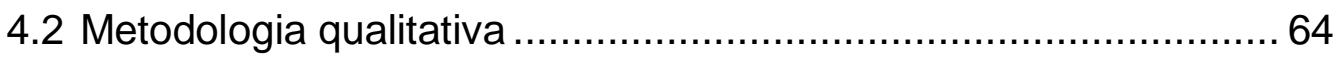

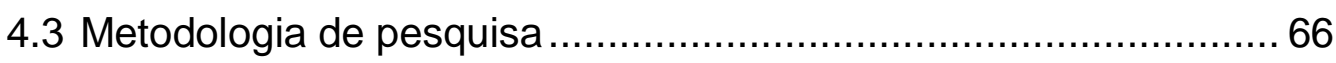

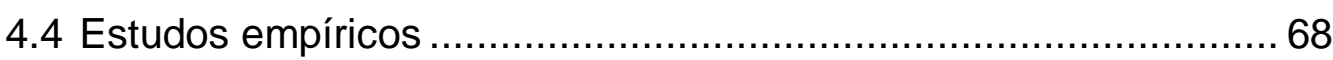

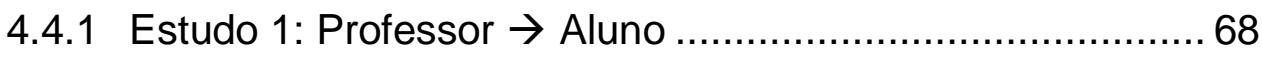

4.4.2 Estudo 2: Bibliotecário $\rightarrow$ Assistente ................................... 74

4.4.3 Estudo 3: Professor $\rightarrow$ Aluno + Padrinho $\rightarrow$ Afilhado .......... 81

4.4.4 Estudo 4: Aluno $\rightarrow$ Professor ............................................. 86 
5 Resultados dos estudos empíricos ................................................. 91

5.1 Visão geral dos estudos ..................................................... 91

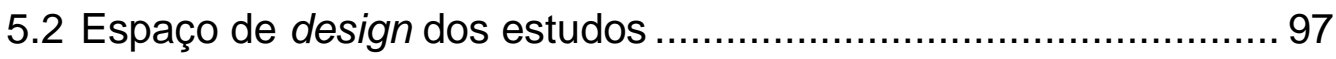

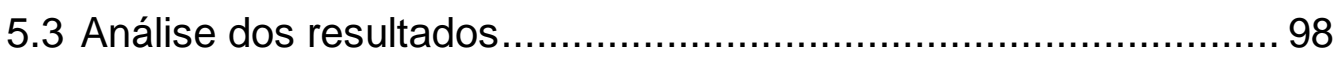

5.3.1 "Este é o meu entendimento de quem você é..." ................ 99

5.3.1.1 Estudo 1............................................................ 100

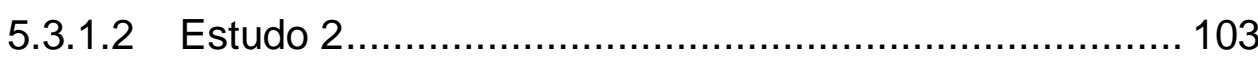

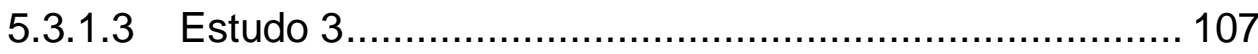

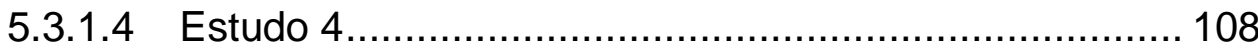

5.3.2 "Este, portanto, é o sistema que projetei para você..."....... 115

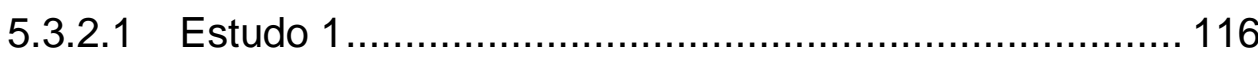

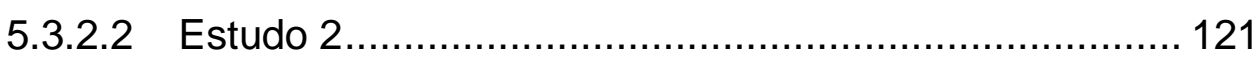

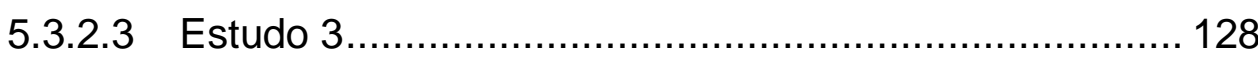

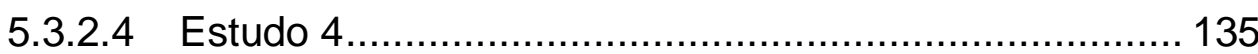

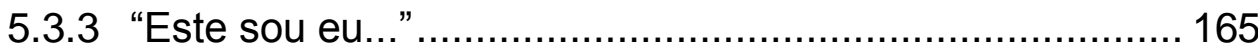

5.3.3.1 Estudo 1......................................................... 167

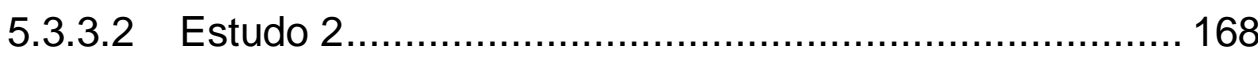

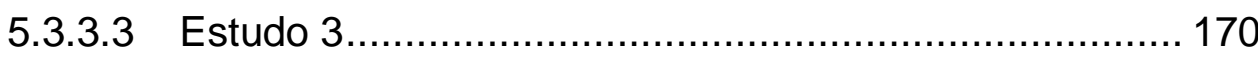

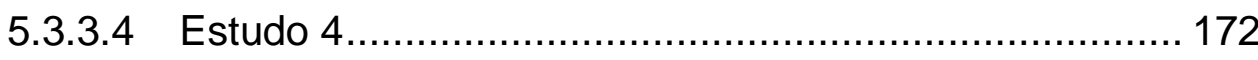

5.3.4 Processo criativo e engenharia semiótica ........................ 187

5.3.4.1 Planejamento da conversa........................................ 188

5.3.4.2 Tempo consumido na criação das conversas .............. 192

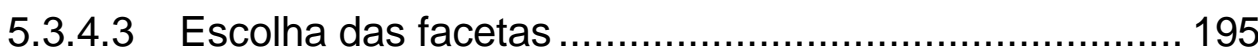

5.3.4.4 Criação de jogos ......................................................... 196

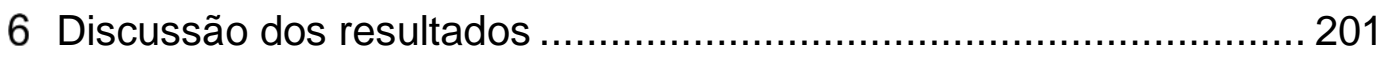

6.1 End-User Semiotic Engineering (EUME) .................................. 201

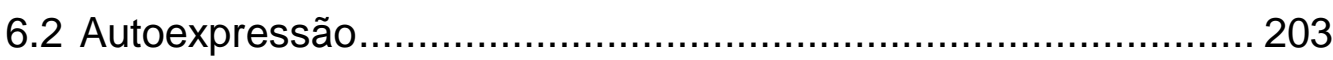

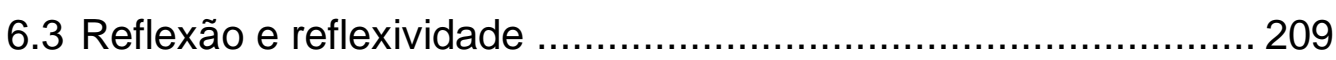

6.4 Um novo olhar em direção à pragmática ................................... 211

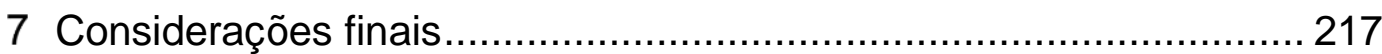

7.1 Contribuições e limitações da pesquisa ..................................... 221

7.2 Oportunidades de trabalhos futuros ........................................... 224

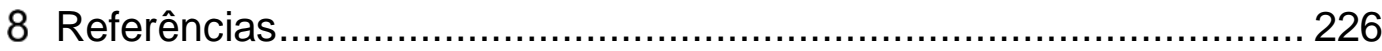

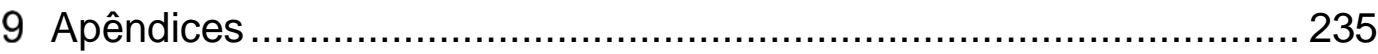

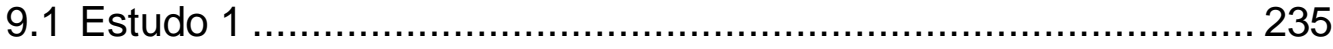




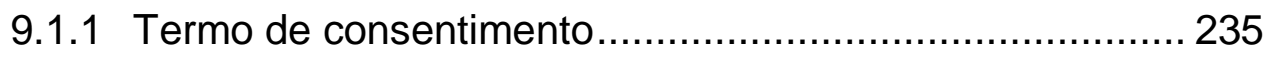

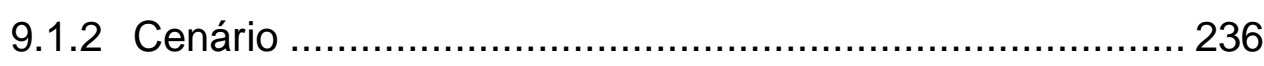

9.1.3 Diálogos de mediação das aulas .......................................236

9.1.3.1 Aula de E1-P1 (Romanos) ....................................... 237

9.1.3.2 Aula de E1-P2 (Órgãos vegetais) .............................. 240

9.1.3.3 Aula de E1-P3 (Triângulo de Pascal) ........................... 249

9.1.3.4 Aula de E1-P4 (Emissão de CO2) ................................ 252

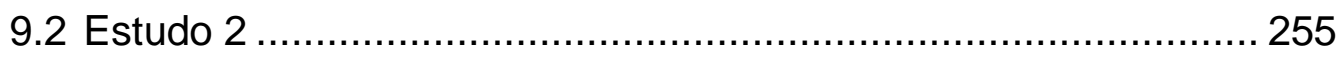

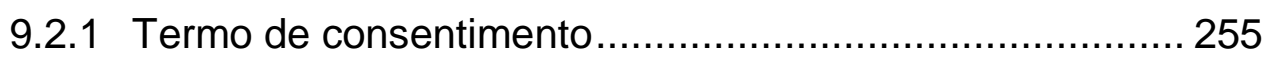

9.2.2 Roteiros de atividade e entrevista com alunos ................ 255

9.2.3 Aula de E2-PC (Aula - Procurar livro biblioteca) .............. 257

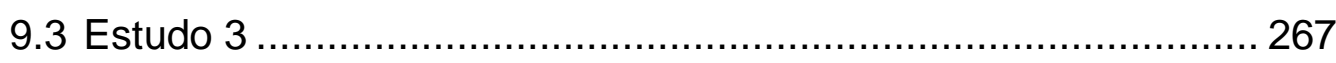

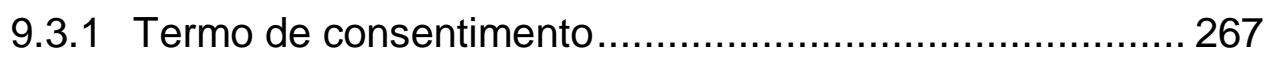

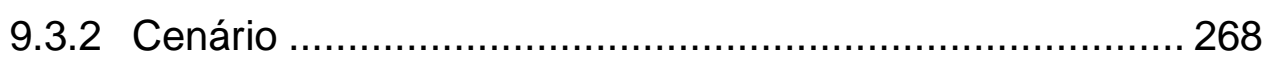

9.3.3 Diálogos de mediação da aula de Física .......................... 269

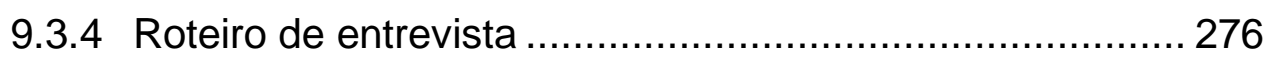

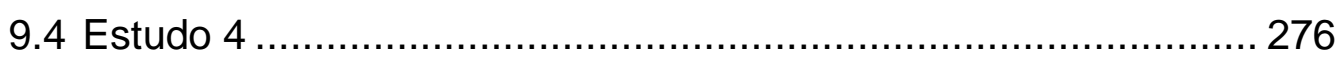

9.4.1 Termo de consentimento dos alunos ................................ 276

9.4.2 Cenário da atividade com os alunos................................. 278

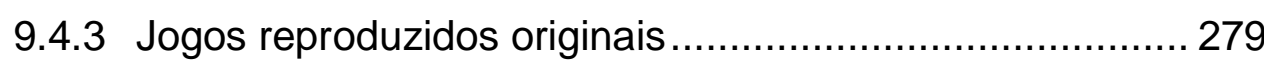

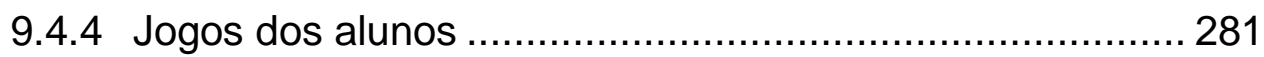

9.4.5 Diálogos de mediação das apresentações ....................... 285

9.4.5.1 Apresentação de E4-PA1 (Veja o meu Sokoban!)......... 285

9.4.5.2 Apresentação de E4-PA2 (Frogger) ............................. 290

9.4.5.3 Apresentação de E4-PA3 (E4-PA3 (Frogger))............... 295

9.4.5.4 Apresentação de E4-PA4 (Space Invaders Game) ...... 299

9.4.5.5 Apresentação de E4-PA5 (My first agentsheets game) 302

9.4.5.6 Apresentação de E4-PA6 (Sokoban (E4-PA6)) ............ 305

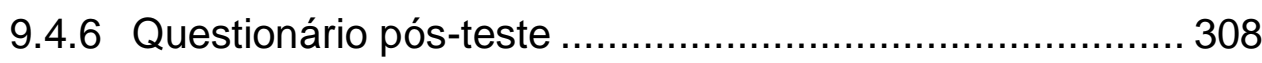

9.4.7 Termo de consentimento do professor ............................... 309

9.4.8 Cenário da atividade com o professor ............................... 310

9.4.9 Perguntas da entrevista com o professor .......................... 311

9.4.10 Nuvem de palavras ..................................................... 311 


\section{Lista de figuras}

Figura 1-1. Esquema conceitual do SideTalk ....................................... 21

Figura 2-1. Comunicação entre designer e usuário ................................ 32

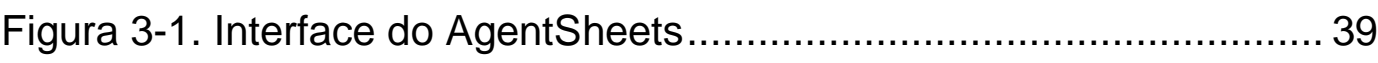

Figura 3-2. Regras de movimento pelas setas .................................... 40

Figura 3-3. "Frogger" no PoliFacets (faceta descrição) .......................... 42

Figura 3-5. Tags do projeto Frogger ..................................................... 43

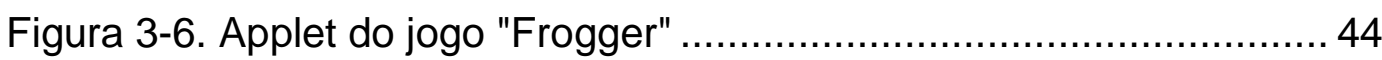

Figura 3-7. Visualização em grade da faceta "planilhas" ......................... 45

Figura 3-8. Diagrama de conexões do agente log................................ 47

Figura 3-9. Tela inicial do WNH (primeira versão) ................................. 49

Figura 3-10. Navegação com WNH (primeira versão) ............................. 49

Figura 3-11. Tela inicial do editor de diálogos ...................................... 50

Figura 3-12. Tela de edição de um diálogo ......................................... 50

Figura 3-13. Tela inicial do SideTalk com lista de conversas .................. 52

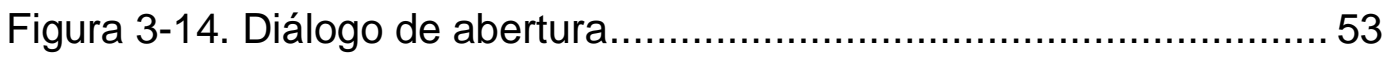

Figura 3-15. Diálogo "Rumo ao Google Imagens" .................................. 53

Figura 3-16. Diálogo "O que você está procurando?".............................. 54

Figura 3-17. Diálogo "Começando a filtrar" ............................................ 54

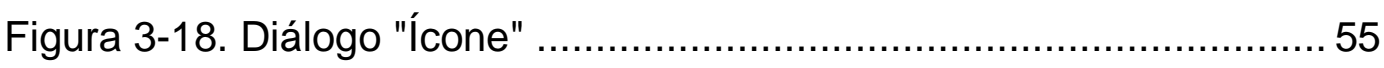

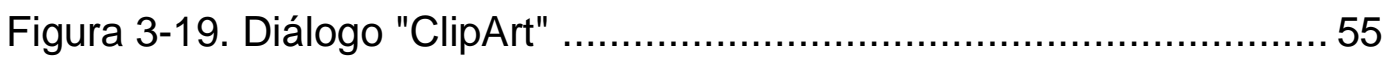

Figura 3-20. Diálogo "Fim da pesquisa" .............................................. 56

Figura 3-21. Edição do script "Pesquisa por ícones" .............................. 58

Figura 3-22. Tela inicial do editor de diálogos do SideTalk ..................... 59

Figura 3-23. Edição do diálogo "O que você está procurando?" ..............59

Figura 3-24. Comunicação entre designers e usuários no SideTalk.........60 60

Figura 3-25. Autoria na comunicação com o SideTalk ............................. 61

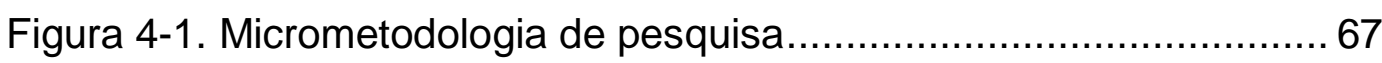

Figura 4-2. Diálogo de abertura da aula "Grupos sanguíneos"................. 70

Figura 4-3. Diálogo 6 da aula "Grupos sanguíneos"................................ 70

Figura 4-4. Diálogo final da aula "Grupos sanguíneos" .......................... 71

Figura 4-5. Planejamento da aula de E1-P3 ........................................ 72

Figura 4-6. Página inicial do site de pesquisa do acervo ......................... 78 
Figura 4-7. Página de resultados (parte superior) ……........................... 79

Figura 4-8. Página de resultados (parte inferior) …………………........ 79

Figura 4-9. Diálogo "Informe a posição inicial" ....................................... 84

Figura 4-10. Apresentando o jogo Paintball no PoliFacets ...................... 89

Figura 5-1. Relacionamento entre os estudos ..................................... 96

Figura 5-2. Autoria nos Estudos 1 e 2 ............................................... 97

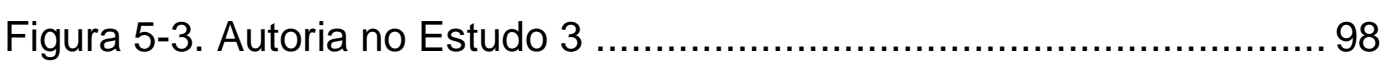

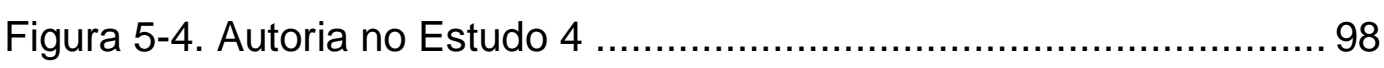

Figura 5-5. Seleção de registros ..................................................... 123

Figura 5-6. Esquema geral da aula ................................................. 128

Figura 5-7. Pontos de retorno de cada participante.............................. 130

Figura 5-8. Sugestões de elementos de interface ............................... 133

Figura 5-9. Agentes principais do jogo de E4-P5 .............................. 138

Figura 5-10. Agentes do Frogger de E4-PA2 ................................... 141

Figura 5-11. Agentes do Frogger original ............................................ 141

Figura 5-12. Agentes com aparências diferentes. ............................... 142

Figura 5-13. Pontos e critérios de originalidade ................................ 148

Figura 5-14. Participantes no continuum de originalidade .................... 149

Figura 5-15. Autoexpressão no PoliFacets........................................ 150

Figura 5-16. Autoexpressão no SideTalk ......................................... 158

Figura 5-17. Frequência de palavras nos diálogos .............................. 177

Figura 5-18. Autoexpressão acumulada nas três ferramentas .............. 186

Figura 5-19. Acumulado de autoexpressão entre os participantes ........ 187

Figura 5-20. Planejamento da aula (E1-P2) ....................................... 189

Figura 5-21. Planejamento da aula (E1-P3) ...................................... 189

Figura 5-22. Tempo $x$ quantidade ................................................. 194

Figura 9-1. Diálogo 1 (abertura) de E1-P1 ...................................... 237

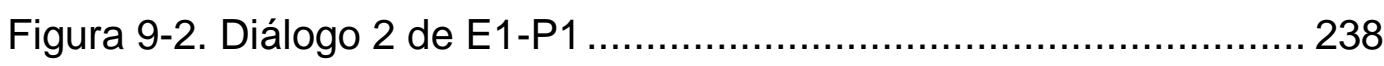

Figura 9-3. Diálogo 3 de E1-P1 ..................................................... 238

Figura 9-4. Diálogo 4 de E1-P1 ..................................................... 239

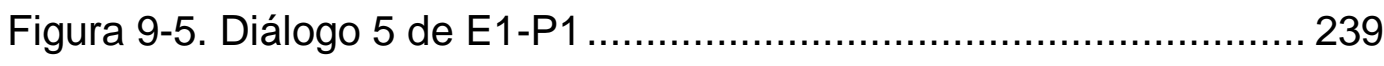

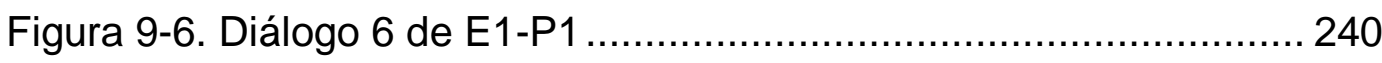

Figura 9-7. Diálogo 1 (abertura) de E1-P2 ....................................... 240

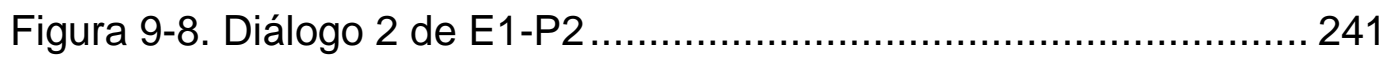

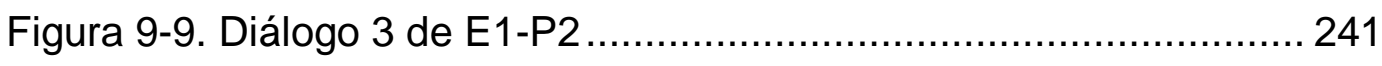




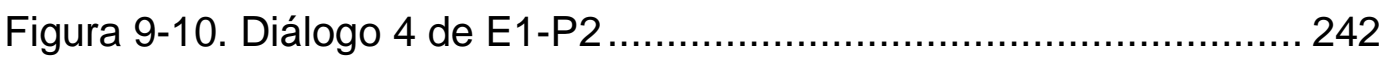

Figura 9-11. Diálogo 5 de E1-P2 …............................................ 242

Figura 9-12. Diálogo 6 de E1-P2 ................................................... 243

Figura 9-13. Diálogo 7 de E1-P2 ................................................... 243

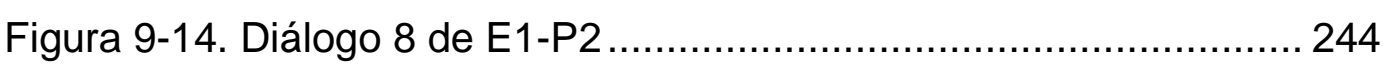

Figura 9-15. Diálogo 9 de E1-P2 .................................................... 244

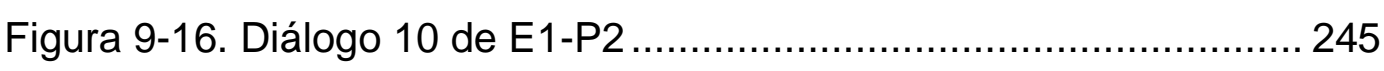

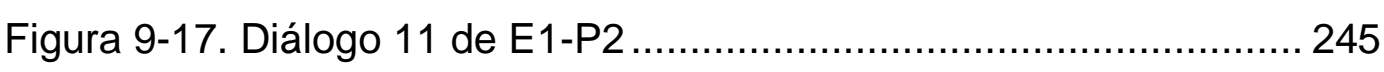

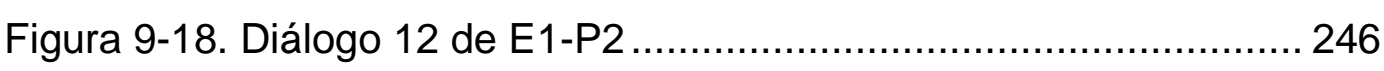

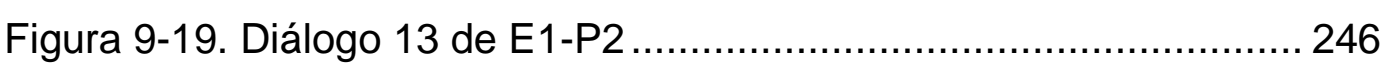

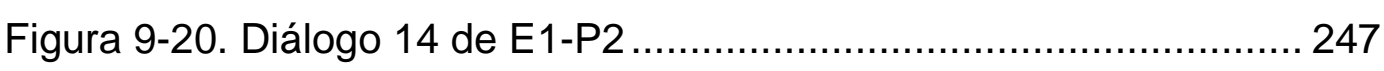

Figura 9-21. Diálogo 15 de E1-P2 ..................................................... 247

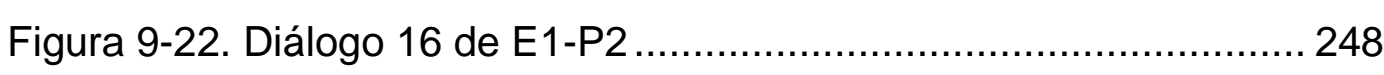

Figura 9-23. Diálogo 17 de E1-P2 ...................................................... 248

Figura 9-24. Diálogo 18 de E1-P2 ...................................................... 249

Figura 9-25. Diálogo 1 (de abertura) de E1-P3................................... 249

Figura 9-26. Diálogo 2 de E1-P3 ....................................................... 250

Figura 9-27. Diálogo 3 de E1-P3 .................................................... 250

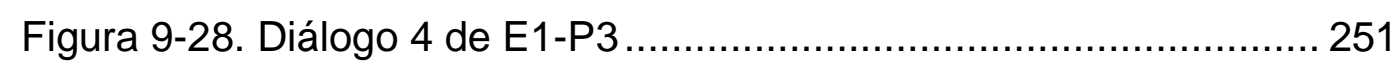

Figura 9-29. Diálogo 5 de E1-P3 ....................................................... 251

Figura 9-30. Diálogo 6 de E1-P3 ................................................... 252

Figura 9-31. Diálogo 1 (abertura) de E1-P4 .................................... 252

Figura 9-32. Diálogo 2 de E1-P4 .................................................. 253

Figura 9-33. Diálogo 3 de E1-P4 .................................................... 253

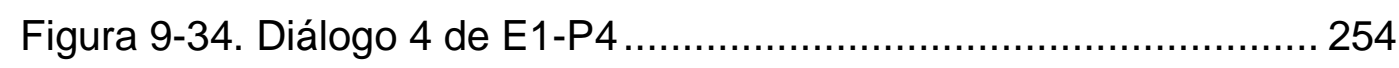

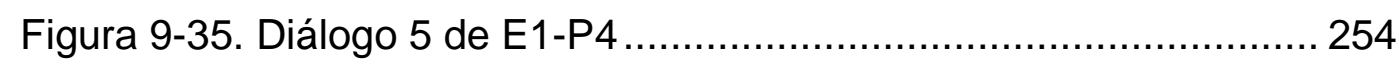

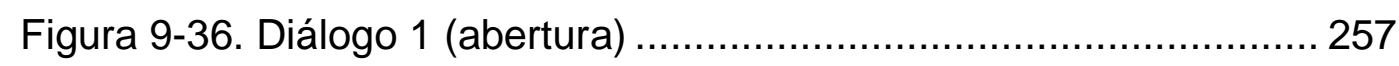

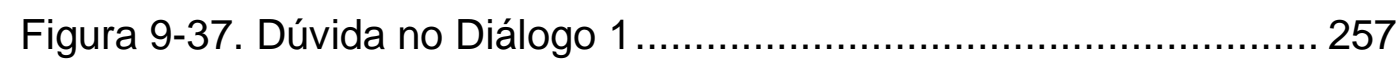

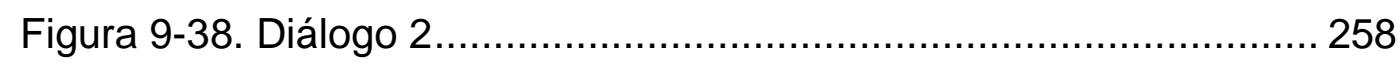

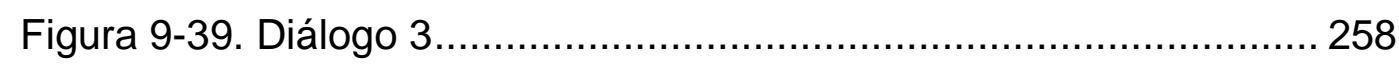

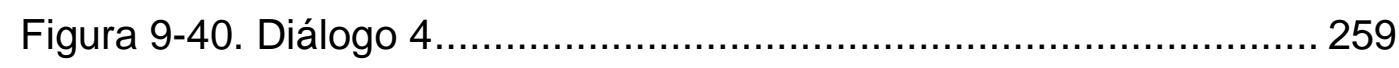

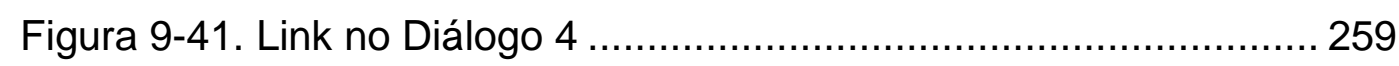

Figura 9-42. Dúvida no Diálogo 4 ................................................... 259

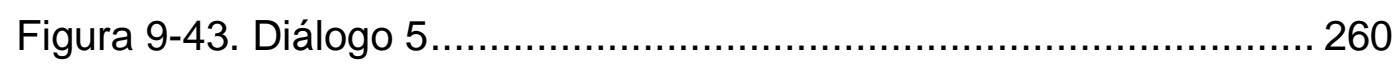

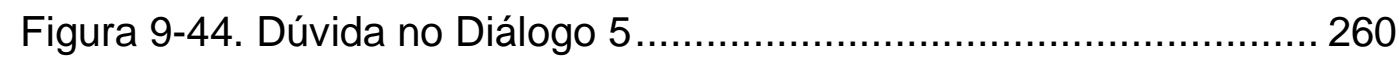




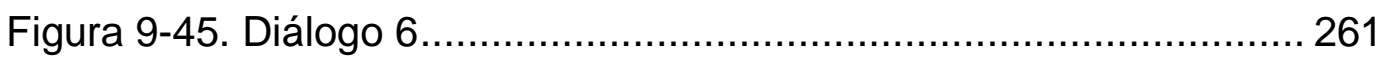

Figura 9-46. Dúvida no Diálogo 6 .................................................. 261

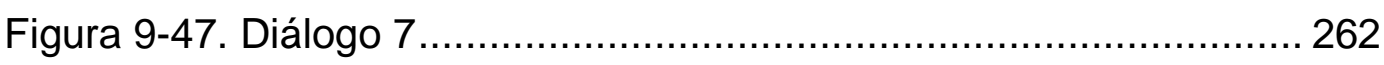

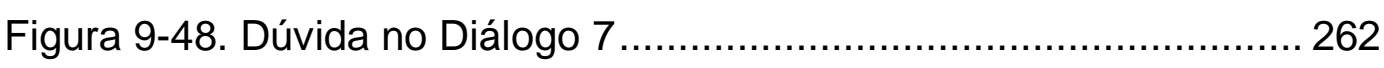

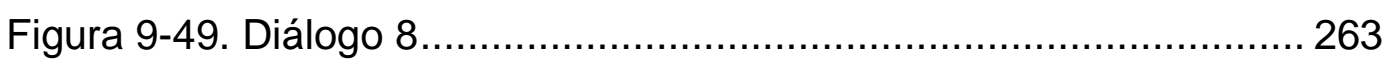

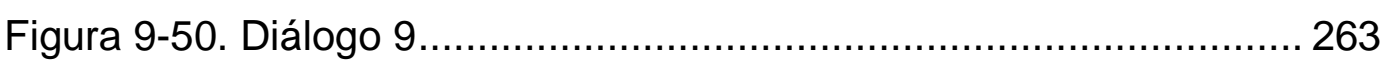

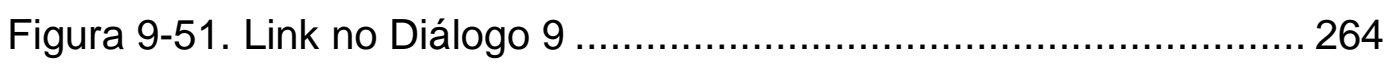

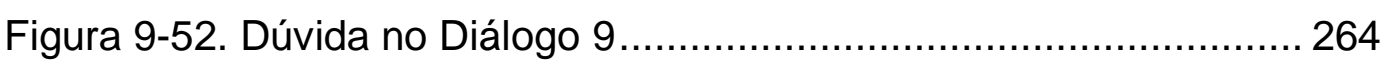

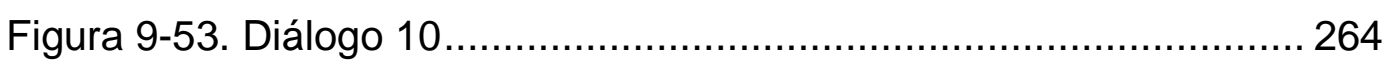

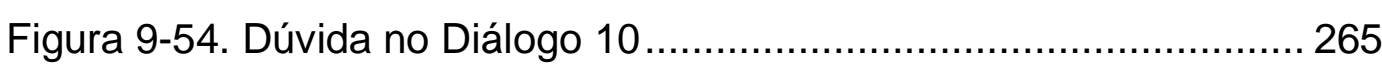

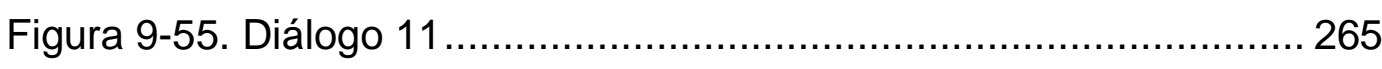

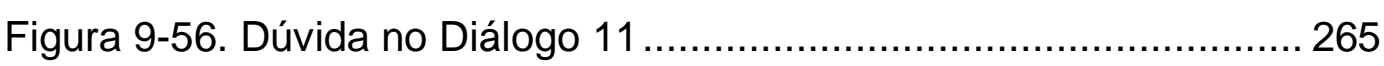

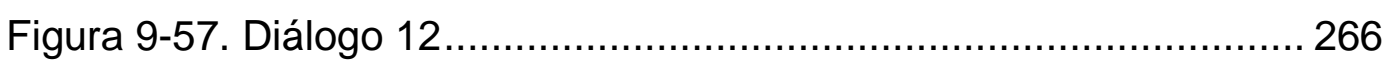

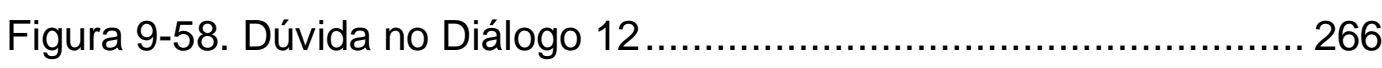

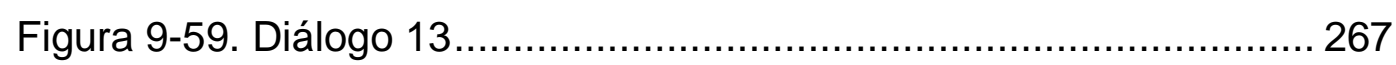

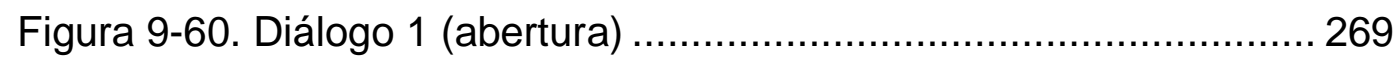

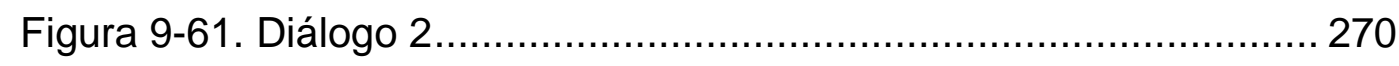

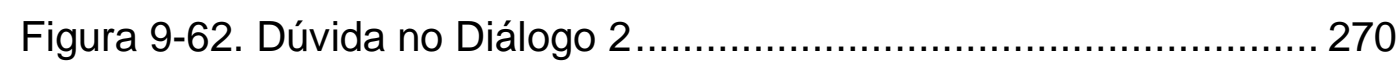

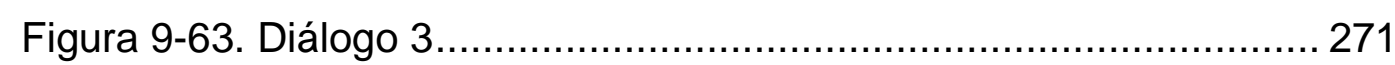

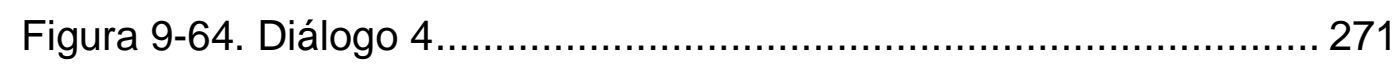

Figura 9-65. Diálogo 5.............................................................. 272

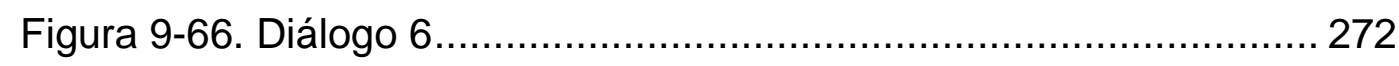

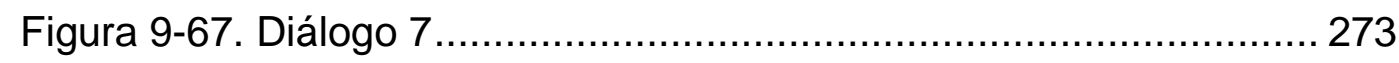

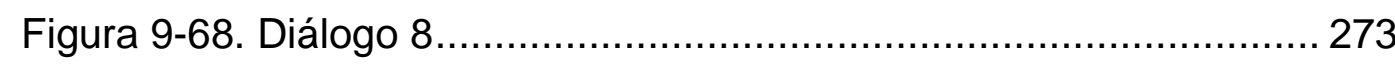

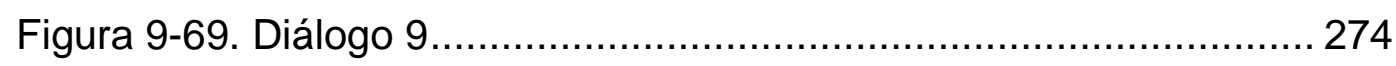

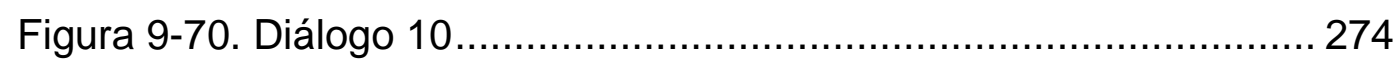

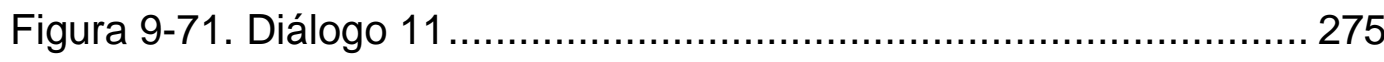

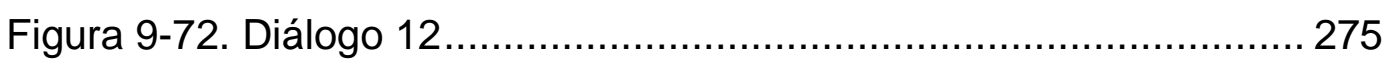

Figura 9-73. Dúvida no Diálogo 12 …............................................. 276

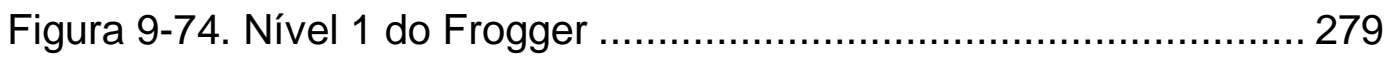

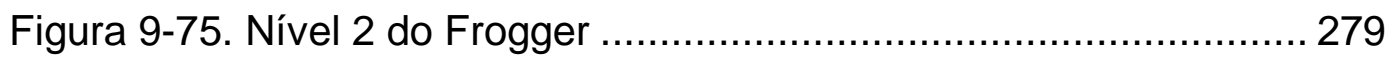

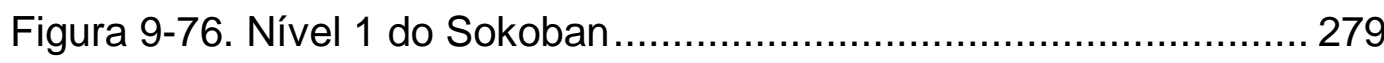

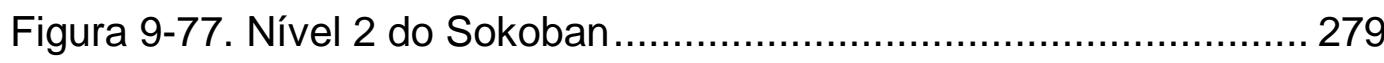

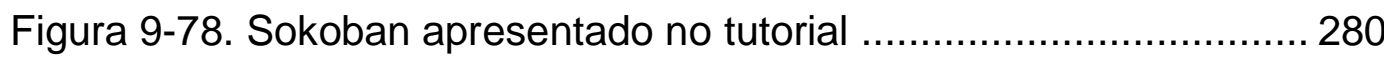

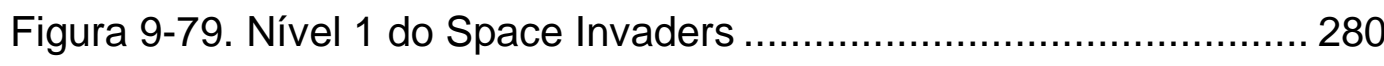


Figura 9-80. Space Invaders apresentado no tutorial .......................... 280

Figura 9-81. Nível 1 do Sokoban de E4-PA1 .................................... 281

Figura 9-82. Nível 2 do Sokoban de E4-PA1 ..................................... 281

Figura 9-83. Nível 3 do Sokoban de E4-PA1 ...................................... 281

Figura 9-84. Nível 1 do Frogger de E4-PA2 ...................................... 282

Figura 9-85. Nível 2 do Frogger de E4-PA2 ...................................... 282

Figura 9-86. Nível 1 do Frogger de E4-PA3 ...................................... 282

Figura 9-87. Nível 2 do Frogger de E4-PA3 ........................................ 283

Figura 9-88. Nível 1 do Space Invaders de E4-P4 .............................. 283

Figura 9-89. Nível 2 do Space Invaders de E4-P4 .............................. 284

Figura 9-90. Nível 1 do jogo de E4-PA5 ............................................. 284

Figura 9-91. Nível 1 do Sokoban de E4-P6 ......................................... 285

Figura 9-92. Nível 2 do Sokoban de E4-P6 ........................................ 285

Figura 9-93. Diálogo 1 (abertura) de E4-PA1 .................................... 285

Figura 9-94. Diálogo 2 de E4-PA1 ................................................... 286

Figura 9-95. Diálogo 3 de E4-PA1 ................................................... 286

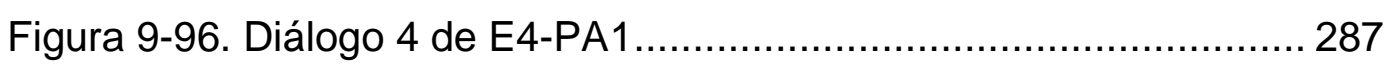

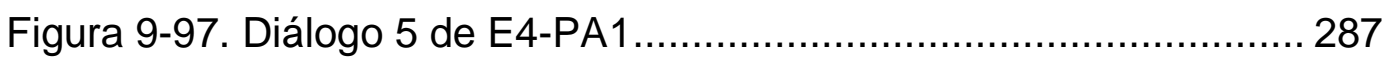

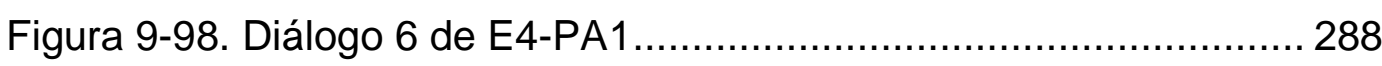

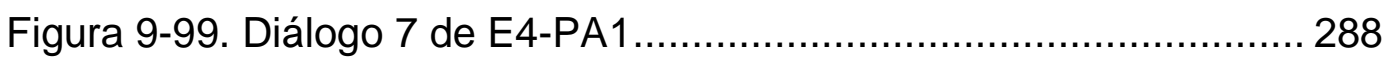

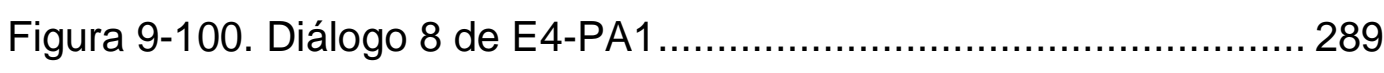

Figura 9-101. Diálogo 9 de E4-PA1................................................ 289

Figura 9-102. Diálogo 1 (abertura) de E4-PA2 .................................. 290

Figura 9-103. Diálogo 2 de E4-PA2 ............................................... 290

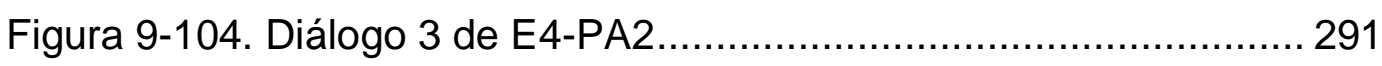

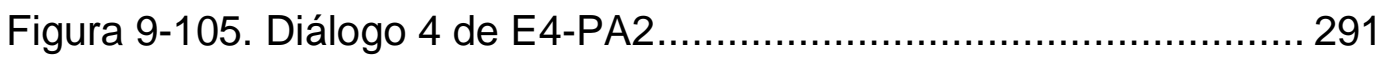

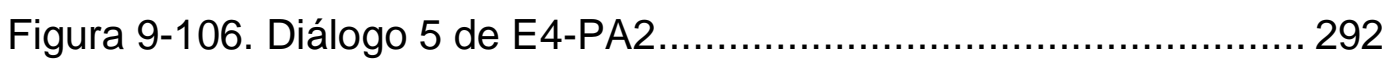

Figura 9-107. Diálogo 6 de E4-PA2 ................................................. 292

Figura 9-108. Diálogo 7 de E4-PA2 ................................................. 293

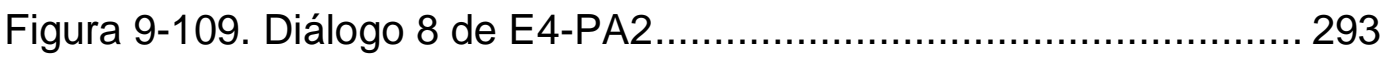

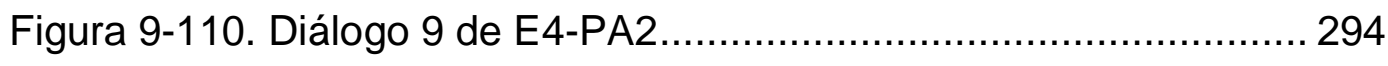

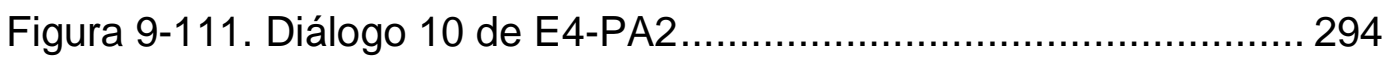

Figura 9-112. Diálogo 1 (abertura) de E4-PA3 ................................... 295

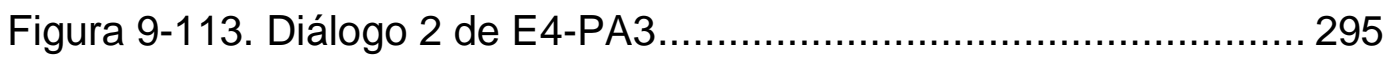

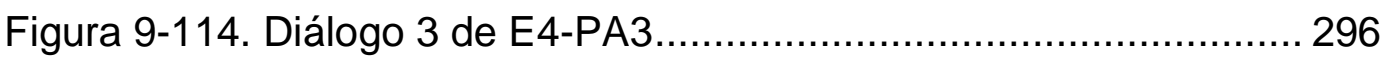




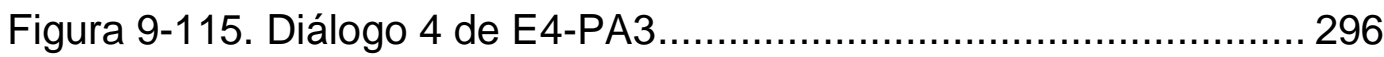

Figura 9-116. Diálogo 5 de E4-PA3............................................... 297

Figura 9-117. Diálogo 6 de E4-PA3.................................................. 297

Figura 9-118. Diálogo 7 de E4-PA3.................................................. 298

Figura 9-119. Diálogo 8 de E4-PA3................................................. 298

Figura 9-120. Diálogo 1 (abertura) de E4-PA4 ................................... 299

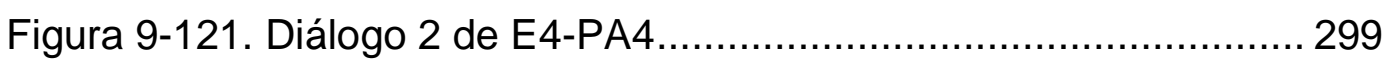

Figura 9-122. Diálogo 3 de E4-PA4.................................................. 300

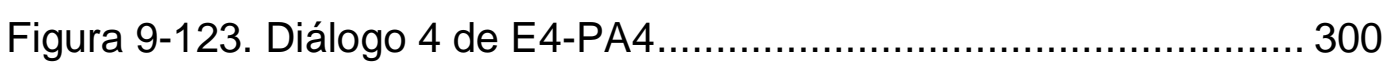

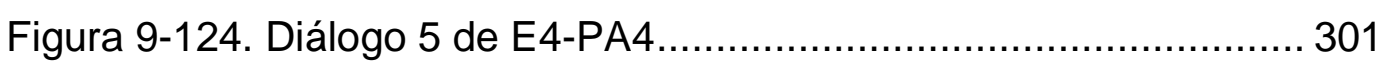

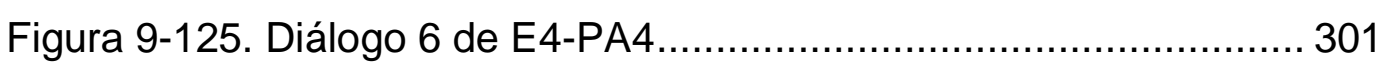

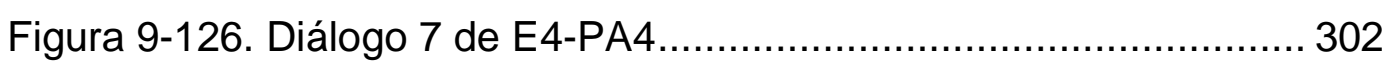

Figura 9-127. Diálogo 1 (abertura) de E4-PA5 …............................... 302

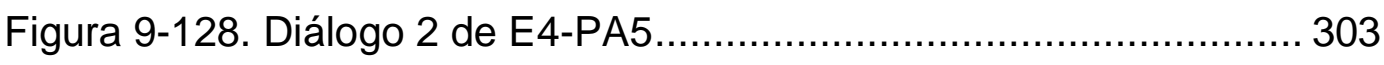

Figura 9-129. Diálogo 3 de E4-PA5.................................................... 303

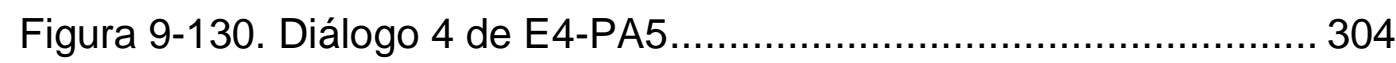

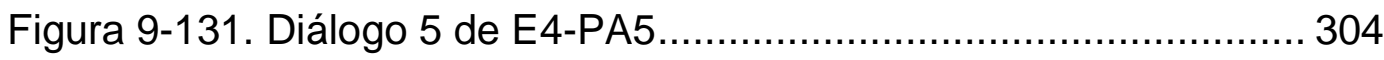

Figura 9-132. Diálogo 1 (abertura) de E4-PA6 ……............................ 305

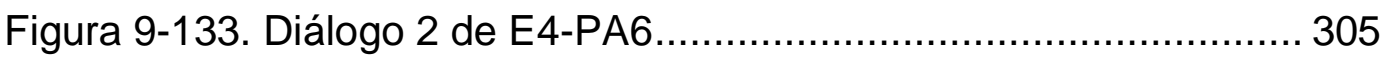

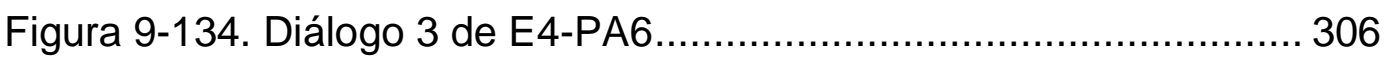

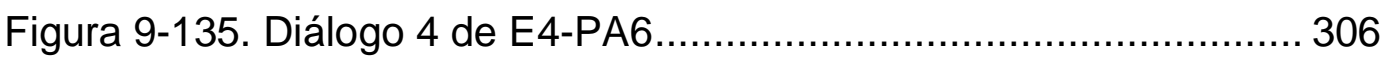

Figura 9-136. Diálogo 5 de E4-PA6 ................................................ 307

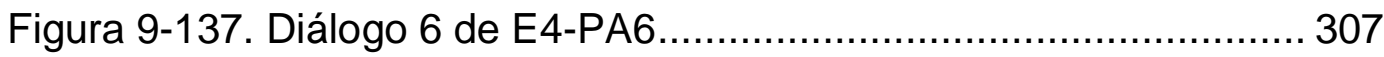

Figura 9-138. Diálogo 7 de E4-PA6 ................................................ 308

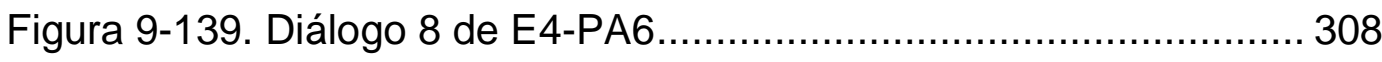




\section{Lista de tabelas}

Tabela 3-1. Formas de representar o código de programas do

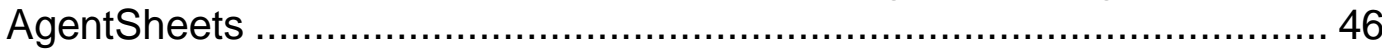

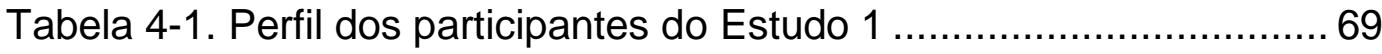

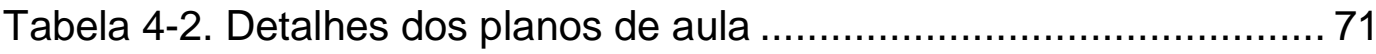

Tabela 4-3. Perfil dos participantes (alunos) ……………………….... 75

Tabela 4-4. Script de base para a aula .............................................. 77

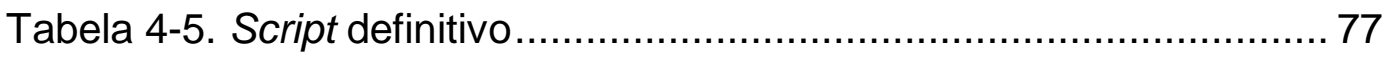

Tabela 4-6. Perfil de participantes do Estudo 3 ................................... 82

Tabela 4-7. Perguntas para configurar os pontos de retorno.................... 85

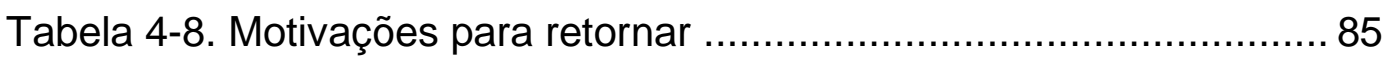

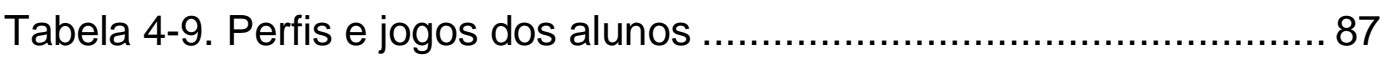

Tabela 5-1. Detalhes sobre os estudos .............................................. 92

Tabela 5-2. Exemplos de modificação textual ..................................... 105

Tabela 5-3. Motivações para retornar (resultado) ................................ 107

Tabela 5-4. Informações dos projetos no PoliFacets............................. 110

Tabela 5-5. Formas de apropriação da aula sugerida .......................... 117

Tabela 5-6. Tamanho das aulas ...................................................... 117

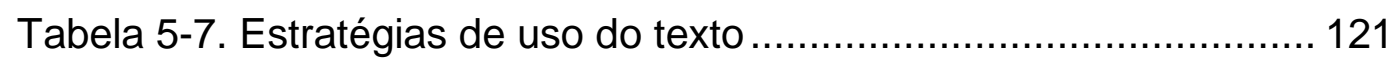

Tabela 5-8. Recursos visuais da aula sobre biblioteca.......................... 122

Tabela 5-9. Conhecimento prévio dos alunos .................................... 127

Tabela 5-10. Novos nomes para agentes e representações ................. 142

Tabela 5-11. Mensagens escritas dos participantes ............................ 147

Tabela 5-12. Indicadores numéricos da produção dos participantes ..... 151

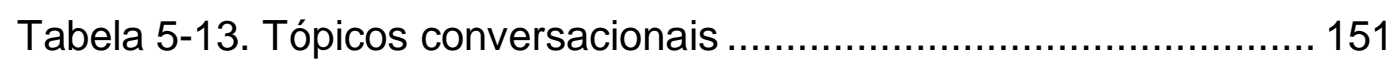

Tabela 5-14. Idioma dos participantes .............................................. 174

Tabela 5-15. Texto de planejamento da conversa................................ 190

Tabela 5-17. Duração (em minutos) das atividades realizadas .............. 193

Tabela 9-1. Lista e frequência de palavras da nuvem de tags............... 312 
Esse é só o começo do fim da nossa vida. Deixa chegar o sonho. Prepara uma avenida, que a gente vai passar... Marcelo Camelo 